\title{
Microtubule Associated Protein 4 Phosphorylation- Induced Epithelial-to-Mesenchymal Transition of Podocyte Leads to Proteinuria in Diabetic Nephropathy
}

\section{Lingfei Li}

Third Military Medical University: Army Medical University

\section{Yanhai Feng}

Third Military Medical University Southwest Hospital

Junhui Zhang

Third Military Medical University Southwest Hospital

\section{Qiong Zhang}

Third Military Medical University Southwest Hospital

Jun Ren

Zhongshan Hospital Fudan University

\section{Cheng Sun}

Third Military Medical University Southwest Hospital

\section{Shujing Li}

Chongqing Medical University

\section{Xia Lei}

Third Military Medical University: Army Medical University

\section{Gaoxing Luo}

Third Military Medical University Southwest Hospital

\section{Jiongyu Hu ( $\nabla$ jiongyuhu@163.com )}

Third Military Medical University Southwest Hospital https://orcid.org/0000-0002-3328-8894

\section{Yuesheng Huang}

Third Military Medical University: Army Medical University

\section{Research Article}

Keywords: MAP4, diabetic nephropathy, proteinuria, epithelial-to-mesenchymal transition, apoptosis

Posted Date: January 10th, 2022

DOI: https://doi.org/10.21203/rs.3.rs-1215351/v1 
License: (c) (i) This work is licensed under a Creative Commons Attribution 4.0 International License. Read Full License 


\section{Abstract \\ Background}

Diabetic nephropathy (DN) involves various structural and functional changes because of chronic glycemic assault and kidney failure. Proteinuria is an early clinical manifestation of DN, but the associated pathogenesis remains elusive. This study aimed to investigate the role of microtubule associated protein 4 (MAP4) phosphorylation (p-MAP4) in proteinuria in DN and its possible mechanisms.

\section{Methods}

In this study, the urine samples of diabetic patients and kidney tissues of streptozotocin (STZ)-induced diabetic mice were obtained to detect changes of microtubule associated protein 4 (MAP4) phosphorylation. A murine model of hyperphosphorylated MAP4 was established to examine the effect of MAP4 phosphorylation in DN. Podocyte was applied to explore changes of kidney phenotypes and potential mechanisms with multiple methods.

\section{Results}

Our results demonstrated elevated content of p-MAP4 in diabetic patients' urine samples, and increased kidney p-MAP4 in streptozocin (STZ)-induced diabetic mice. Moreover, p-MAP4 triggered proteinuria with aging in mice, and induced epithelial-to-mesenchymal transition (EMT) and apoptosis in podocytes. Additionally, p-MAP4 mice were much more susceptible to STZ treatment and showed robust DN pathology as compared to wild-type mice. In vitro study revealed high glucose $(\mathrm{HG})$ triggered elevation of p-MAP4, rearrangement of microtubules and F-actin filaments with enhanced cell permeability, accompanied with dedifferentiation and apoptosis of podocytes. These effects were significantly reinforced by MAP4 hyperphosphorylation, and were rectified by MAP4 dephosphorylation. Notably, pretreatment of p38/MAPK inhibitor SB203580 reinstated all HG-induced pathological alterations.

\section{Conclusions}

The findings indicated a novel role for p-MAP4 in causing proteinuria in DN. Our results indicated the therapeutic potential of MAP4 in protecting against proteinuria and related diseases.

\section{Background}

Diabetic nephropathy (DN) is the most common form of complication for renal replacement therapy and prompts end-stage renal failure in diabetes mellitus (Locatelli et al., 2004, Gallagher and Suckling, 2016). DN involves a series of structural and functional changes when subjected to chronic glycemic assault 
(Czajka et al., 2015). Profound proteinuria, which heralds a gradual deterioration in glomerular function, commonly occurs in the early stage of DN (Zurbig et al., 2012). The magnitude of proteinuria has been commonly employed to assess DN severity. Despite considerable advances in understanding the mechanisms governing the permselective properties of glomerular capillary wall, pathogenesis of proteinuria remains unclear in $\mathrm{DN}$.

Ample evidence has depicted that podocyte slit diaphragm and foot processes are important barriers of glomerular filter, and breakage of the barrier integrity is a leading cause in the progression of proteinuria (Rask-Madsen and King, 2010). Among various available pathogenic theories for podocyte dysfunction in DN, epithelial-to-mesenchymal transition (EMT) is one of the responses that occurs in certain kidney diseases (Kang et al., 2010). When subjected to EMT, cytoskeleton of podocytes rearranged, destroyed their cell junctions and basal-apical polarity, altered their interaction with extracellular matrix, and obtained mesenchymal features, such as invasiveness and elevated motility (Ying and Wu, 2017).

Microtubule-associated proteins (MAPs) are primarily recognized as skeletal proteins which bind to tubulins to promote their assembly. The family of MAPs encompass tau, MAP2 and MAP4. MAP4 is widely expressed in non-neural cells and plays an essential role in maintaining microtubule (MT) dynamics (Illenberger et al., 1996, Drewes et al., 1998, Hu et al., 2010, Li et al., 2015). Additionally, MTMAPs binding is modulated by MAPs phosphorylation which leads to dissociation of MAPs from MT and instability of MT (Ebneth et al., 1999, Hu et al., 2014). We and others have reported that the human MAP4 (S768 and S787) phosphorylation is crucial in governing its detachment from MTs, and mitogenactivated protein kinase kinase 6 (MKK6)/p38 MAPK serves as the upstream signal for hypoxia-mediated MAP4 phosphorylation and followed by MT depolymerizaiton (Raingeaud et al., 1996, Kitazawa et al., 2000, Hu et al., 2010).

In current study, we firstly demonstrated increased MAP4 phosphorylation in kidneys of streptozotocin (STZ)-induced diabetic mice. Subsequently, we constructed mouse models to mimick MAP4 phosphorylation in specific-sites (S737 and S760). Somewhat to our surprise, MAP4 phosphorylation initiated proteinuria with aging, accompanied with incremental EMT and apoptosis of podocytes. In addition, MAP4 phosphorylation mice were much more susceptible to STZ treatment and showed robust DN pathology as compared to wild-type mice. In vitro, F-actin and MT rearrangement induced by MAP4 phosphorylation was deemed potential mechanisms of podocyte EMT, promoting to the development of proteinuria. Altogether, the findings favor a novel role for MAP4 phosphorylation in contributing to proteinuria in $\mathrm{DN}$, indicating its therapeutic potential in the management of proteinuria and related diseases.

\section{Materials And Methods}

\section{Ethics statement of patient urine samples}

The Ethics Committee of Southwest Hospital, Third Military Medical University (Army Medical University) approved the present study (approval number: KY2020085). Prior to be included in the study, all 
participants signed relevant informed consent. Participants were identified using a number, not their name. It was conducted in adherence with the Declaration of Helsinki. All methods were carried out in accordance with the institutional guidelines of the ethics committee at Southwest Hospital, Third Military Medical University (Army Medical University) (Chongqing, China).

\section{Urine sample collection}

Eight urine samples of patients with clinically diagnosed diabetes were collected from the inpatients who signed a consent form. These inpatients received formal treatment at Endocrinology Department, Southwest Hospital, Third Military Medical University (Army Medical University). The samples used were the urine left over from routine urine collections, which were midstream samples. All samples were assigned a study number that connected them to clinical information from the chart to deidentify them. The samples were used for ELISA analysis.

\section{Ethics statement of animal study}

All animal experiments were implemented in accordance with the Guide for the Care and Use of Laboratory Animals announced by the US National Institutes of Health (NIH Publication, 8th Edition, 2011) and were granted permission by the Animal Experiment Ethics Committee of the Army Medical University (the Third Military Medical University).

\section{Animal study}

The knock-in mice that mimicked MAP4 hyperphosphorylation in specific-sites (S737 and S760, MAP4 KI mice) were performed as previously described (Li et al., 2018). Wild-type (WT) littermates were used as controls for experiments with corresponded MAP4 KI male or female mice. The age of mice ranged from 10-74 weeks. The C57BL/6J or MAP4 KI mice (10 to 74 weeks) applied for STZ (Cat\# S0130, Sigma, Darmstadt, Germany)-induced diabetes were starved for 8-12h and then injected with a single intraperitoneal dose of STZ in sodium citrate buffer at $150 \mathrm{mg} / \mathrm{kg}$ body weight. Mice with a glucose concentration exceeding $16.7 \mathrm{mmol} / \mathrm{L}$ were considered diabetic. Mice were anesthetized with pentobarbital sodium $(1 \%, 50 \mathrm{mg} / \mathrm{kg})$ by intraperitoneal injection and sacrificed 5 weeks after STZ injection. Experiments involving animals were performed in accordance with United Kingdom Home Office and European Union guidelines and were approved by the Animal Care Centre of the Third Military Medical University.

\section{Biochemical tests of urine and blood samples}

Mouse random urine was collected and centrifuged, and blood samples were collected by retro-orbital puncture from anesthetized animals after fasting 8-12h. Urine albumin, urine creatinine (CR), serum levels of urea nitrogen (UN), CR and cystatin c (Cys-c) were determined using commercial kits.

\section{Histology and ultrastructural pathology}


Renal tissue was cut and fixed with $10 \%$ formalin, embedded in paraffin or made in frozen, and sectioned at 5- $\mu \mathrm{m}$ thickness for routine histopathology or Masson's trichrome staining. Six serial sections from each mouse were used to measure the glomerular volume: the volume of the glomerular was calculated as previously described (Najafian et al., 2002, Chen et al., 2017). For transmission electron microscope (TEM) observation, tissues of renal cortical were fixed in $2.5 \%$ glutaraldehyde followed by dehydration, vibratome sliced and recut on a microtome and stained with uranyl acetate and lead citrate overnight. The degree of foot process fusion was determined by measuring the number of junctions per 1 micron length of glomerular basement membrane (GBM) as described previously (Asanuma et al., 2005).

\section{Podocyte culture and treatment}

Conditioned immortalized murine podocytes were obtained and cultured as our study described previously (Shankland et al., 2007, Chen et al., 2017). Cells were used for experiments as stated. After transfection of adenovirus overexpressing MAP4(Ala), MAP4(Glu), MKK6(Glu), or CMV-null for 36h, the cells were treated with $5.6 \mathrm{mM}$ D-glucose, $5.6 \mathrm{mM}$ D-glucose $+25 \mathrm{mM}$ mannitol or $30 \mathrm{mM}$ D-glucose for 48h. During the stimulation, SB203580 (Calbiochem Cat\# 559389, $10 \mu \mathrm{M}$, Darmstadt, Germany) was applied to pretreat cells for $1 \mathrm{~h}$ prior to glucose treatment.

\section{Adenovirus construction}

Site-directed mutagenesis of MAP4 and MKK6, construction and transduction of recombinant adenoviruses overexpression MAP4(Glu), MAP4(Ala) and MKK6(Glu) were performed as described previous (Hu et al., 2014, Li et al., 2015, Li et al., 2018). MAP4(Ala) mimicked MAP4 (S737 and S760) dephosphorylation, MAP4(Glu) mimicked MAP4 (S737 and S760) phosphorylation and MKK6(Glu) was an upstream kinase of p38/MAPK, which was consistently activated p38/MAPK.

\section{Immunofluorescence}

Podocytes or frozen sections were used as described previously in our reports (Li et al., 2018). Antibodies: a-tubulin (Proteintech Group Cat\# 11224-1-AP, RRID:AB_2210206, Rosemont, IL, USA), Phalloidin (Thermo Fisher Scientific Cat\# A12379, RRID:AB_2315147, Waltham, MA, USA), Wilms Tumor protein (WT-1) (Abcam Cat\# ab89901, RRID:AB_2043201, Cambridge, Cambridgeshire, United Kingdom), fibroblastspecific protein 1 (FSP1) (Abcam Cat\# ab197896, RRID:AB_2728774), Nephrin (R and D Systems Cat\# AF3159, RRID:AB_2155023, Minneapolis, MN, USA), Desmin (R and D Systems Cat\# AF3844, RRID:AB_2092419).

\section{Apoptosis assay}

In Situ Cell Death Detection Kit, fluorescein (Cat\# 11684795910, Roche, Basel, Switzerland) were used for apoptosis assay of frozen kidney sections (WT-1 and terminal deoxynucleotidyl transferase-mediated dUTP nick-end labeling (TUNEL) co-staining) or podocytes.

\section{Cell proliferation assay}


Cell proliferation determined by 5-ethynyl-2'-deoxyuridine (EdU) assay was carried out using the Click-iT ${ }^{\circledR}$ EdU imaging detecting kit according to the manufacturer's instructions (Cat\# BCK488-IV-IM-S, Sigma).

\section{Western blot (WB) analysis}

Renal tissue samples and podocytes were used for WB experiments following our previously described procedures (Li et al., 2018). Antibodies: WT-1 (Abcam Cat\# ab89901, RRID:AB_2043201), FSP1 (Abcam Cat\# ab197896, RRID:AB_2728774), Nephrin (R and D Systems Cat\# AF3159, RRID:AB_2155023), Desmin (R and D Systems Cat\# AF3844, RRID:AB_2092419), GAPDH (Proteintech Group Cat\# 60004-1-Ig, RRID:AB_2107436), MAP4 (Bethyl Cat\# A301-489A, RRID:AB_999616, Montgomery, Texas, USA), p38 MAPK (P38) (Cell Signaling Technology Cat\# 9212, RRID:AB_330713, Danvers, MA, USA), Phospho-p38 MAPK (p-P38) (Cell Signaling Technology Cat\# 9211, RRID:AB_331641). Rabbit polyclonal antibodies against p-MAP4 (S787) and p-MAP4 (S737) were made in-house and validated as reported previously (Li et al., 2018).

\section{Enzyme-Linked Immunosorbent Assay (ELISA)}

Specific ELISA kits were purchased from the Mlbio Group to detect the concentrations of MAP4 and pMAP4 in urine samples. Detection procedures were conducted according to the manufacturer's instructions.

\section{Measurement of transepithelial electric resistance (TER)}

Cellular barrier properties were assessed using assessment of TER across confluent podocytes with Millicell-ERS (MERS00002, Millipore, Darmstadt, Germany) (Li et al., 2015). A 24-well transwell system (Greiner Bio-One, 0.4-mm pore size, 6.5-mm diameter, transparent, Costar) was inserted into the 24-well plate. Podocytes were plated in transwell chambers. The volumes of culture medium were 100 and $600 \mu l$ in the upper and lower compartments, respectively, in which cells were allowed to grow for 2 days (considered day 0 ). The resistance (R sample) was measured, the value for the blank well represented the blank resistance ( $R$ blank). The following calculation was performed: epithelial monolayer resistance $=(R$ sample-R blank) * effective membrane area (the area of a 24-well transwell chamber is $0.336 \mathrm{~cm}^{2}$ ).

Measurement of the permeability to fluorescein isothiocyanate (FITC)-dextran Cells were grown on transwell compartments as above described. The permeability was measured by the addition of FITCdextran (40 kDa, Sigma-Aldrich) for $1 \mathrm{~h}$ (Li et al., 2015).

\section{Retinal angiography imaging}

Angiography imaging was obtained with spectralis HRA + OCT (Heidelberg Engineering, Heidelberg, Germany). In brief, pupils were dilated using topical tropicamide phenylephrine eye drop (Mydrin-P, Santen. Oy, Japan). Mice were anesthetized as described above. Fluorescein sodium salt ( $5 \mathrm{ml}: 0.5 \mathrm{~g}$, Alcon Laboratories, Freiburg, Germany) was injected intraperitoneally as fluorescent tracer. Imaging was 
obtained at 488-nm absorption and 495-nm emission using a $55^{\circ}$ lens. Images of the central retina were taken, with the optic nerve located in the center of the image.

\section{Statistical analysis}

All values are means \pm SEM (for bar graphs) or SD (for univariate scatter plots). Statistical differences between groups were assessed using two-tailed Student's $t$ test or one-way analysis of variance (ANOVA) post hoc tests, as appropriate. For all studies, values of $P<0.05$ were considered statistically significant.

\section{Results}

\section{MAP4 phosphorylation increased in urine samples of diabetic patients}

To identify the variations of MAP4 and its phosphorylation in patients with clinically diagnosed diabetes, we measured them through ELISA after urine of diabetic patients was collected. As indicated in Figure 1, a significant up-regulation of MAP4 phosphorylation was observed in urine $(p<0.05)$, and simultaneously, no obvious variation of total MAP4 protein was noted $(p>0.05)$. This result demonstrated that increased MAP4 phosphorylation existed in urine of diabetic patients.

\section{Nephritic MAP4 hyperphosphorylation along with proteinuria in DN}

A significant reduction in body weight and random blood glucose were observed in STZ experimental diabetic mice compared with the WT littermates (Figure. 2A-B). Using WB analysis, phosphorylation levels of MAP4 at S737 and S760 were significantly elevated in renal tissues from STZ-induced diabetic mice compared with WT mice (Figure. 2C-D). Although little changes were noted in serum CR, serum UN and serum Cys-c between the two groups (Figure. $2 \mathrm{~F}-\mathrm{H}$ ), the early indicator of renal injury-urine albumin/CR ratio (UACR) was significantly elevated in STZ-induced diabetic mice (Figure. 2E). An increase in glomerular volume was evident using the haematoxylin-eosin (HE) staining (Figure. $2 \mathrm{I}$ and $\mathrm{J}$ ), and Masson's trichrome staining displayed basement membrane thickening, collagen accumulation and a rise in mesangial matrix (Figure. 2K). Ultrastructure of glomerular filtration barrier was further examined using TEM. STZ treatment induced foot process fusion (as depicted in enlarged view in Figure. 2M), associated with severely damaged cytoplasmic structures of podocytes (Figure. 2M). To assess the degree of foot process fusion, the number of junctions per $\mu \mathrm{m}$ GBM was determined (Figure. $2 \mathrm{~L}$ ). WT mice possess much more junctions per $\mu \mathrm{m}$ GBM than STZ mice, suggesting enhanced foot processes effacement in STZ mice. We further examined the effect of STZ on epithelial and mesenchymal markers in podocytes. As shown in Figure. $2 \mathrm{~N}$ and $\mathrm{O}$, immunofluorescence staining revealed that STZ challenge inhibited the levels of epithelial markers, including Nephrin and WT-1, while promoting mesenchymal markers expression, including FSP-1 and Desmin. In addition, WT-1, a marker of podocyte, and TUNEL co-staining revealed increased podocyte apoptosis (white arrows) in glomeruli (Figure. 2P and Q). Little difference was noted in cell proliferation using EdU staining between STZ and WT group (Figure. 2R and S). Taken together, these data indicated that STZ treatment led to overt albuminuria, podocyte dedifferentiation and apoptosis in glomeruli. 


\section{MAP4 hyperphosphorylation mutation leads to proteinuria and nephropathy reminiscent of diabetic kidney disease in MAP4 KI mice with or without STZ stimulation.}

To elucidate potential pathological role of MAP4 phosphorylation in kidney disease, renal function and morphology were examined in MAP4 KI mice. MAP4 KI mice showed increased phosphorylation levels of MAP4 at S737 and S760 in renal tissues compared with those from WT normal mice (Figure. 3A-B). Little changes in serum levels were noted for CR, UN and Cys-c between the two groups (up to 70-74w) (Figure. 3D-F), while UACR was drastically elevated in MAP4 KI mice at 70-74 weeks of age (Figure. 3C). Interestingly, a decrease in volume of glomerular volume in MAP4 KI mice was detected at the age of 7074 weeks using HE staining (Figure. 3G-H). An increase in basement membrane thickening and collagen accumulation was observed by Masson Trichrome staining at the ages of 30-34w (Figure. 3G). Using TEM evaluation, widening of slit membranes was detected in podocytes (enlarged view in Figure. $3 \mathrm{~J}$ ) and WT mice displayed much more junctions per $\mu \mathrm{m}$ GBM compared with MAP4 KI mice at the age of 30-34 and 70-74 weeks (Figure. 3I), suggesting increased foot processes effacement in MAP4 KI mice.

Profound thickening of basement membrane and podocytes detachment were also seen in MAP4 KI mice at the age of 30-34 and 70-74 weeks (black arrowheads in Figure. 3J). Co-staining of WT-1 and TUNEL revealed elevated apoptosis of podocytes (white arrowheads) at 30-34 and 70-74 weeks of age in MAP4 $\mathrm{KI}$ mice as compared to WT littermates (Figure. 3K-L). There was little difference between MAP4 KI and WT groups in cell proliferation using EdU staining (Figure. 3M-N). Immunofluorescence staining revealed decreased expressions of epithelial markers, such as Nephrin and WT-1, and elevated expressions of mesenchymal indicators, including FSP1 and Desmin in glomeruli of 30-34 and 70-74 week-old MAP4 KI mice, indicating podocyte dedifferentiation (Figure. 4A-B). Given that retinopathy is an important microvascular complication of diabetes, concurrent with DN, we further investigated retinopathy in MAP4 $\mathrm{KI}$ mice. Our results revealed normal retinal vasculature in WT mice at 70-74 weeks of age (Figure. $4 \mathrm{C} \mathrm{a}$, b). The data shown in Figure. 4C e-f exhibited microscopic aneurysms with balloon-like structures budding off capillary walls in retinal vessels from 70-74w mutant mice, similar to STZ-induced diabetic model (Figure. 4C c-d). MAP4 KI aggravated STZ-induced microscopic aneurysms (Figure. 4C g-h).

To detect the kidney phenotypes of WT and MAP4 KI mice after STZ treatment, renal function and morphology were further examined at the age of 10-14 weeks between the two mice. The results demonstrated that MAP4 KI STZ mice displayed deteriorated pathological kidney changes as compared to WT STZ mice, as represented by increased UACR, EMT, foot process fusion and basement membrane thickening (Figure 5A, 5E-H). However, little difference was observed in serum UN, CR and Cys-c between the two groups (Figure 5B-D).

Taken together, the appearance of proteinuria, foot process fusion, EMT or apoptosis of podocytes suggested disrupted integrity of glomerular filtration barrier in MAP4 KI mice reminiscent of the typical DN pathology, and STZ treatment worsen such DN pathology in MAP4 KI mice as compared to WT littermates. Simultaneous occurrence of retinopathy in MAP4 KI mice suggested the nephropathy may attribute to the onset of microvascular disease in diabetes. 


\section{MAP4 phosphorylation induced EMT, cytoskeleton rearrangement and apoptosis in podocytes}

Earlier evidence suggested that podocytes may play an essential role in defective glomerular filtration barrier triggered by MAP4 hyperphosphorylation. To discern the impact of MAP4 phosphorylation in podocytes, adenovirus carrying MAP4(Ala) or MAP4(Glu) was applied to podocytes under high glucose (HG) challenge. WB analysis was performed to confirm MAP4(Glu) or MAP4(Ala) transfection (Figure. 6A). HG treatment down-regulated WT-1 and Nephrin, while up-regulating FSP1 and Desmin. Transfection with MAP4(Glu) and MAP4(Ala) aggravated and alleviated HG-induced dedifferentiation in podocytes, respectively (Figure. 6B-C). Immunofluorescence staining revealed similar trend for WT-1, Nephrin, FSP1 and Desmin in podocytes (Figure. 6D-E).

The effect of MAP4 phosphorylation on barrier function of podocytes was also examined. Permeability of monolayer podocyte was monitored by evaluating the TER or FITC-conjugated dextran influx across cells. HG treatment induced decreased TER while increased dextran leakage. MAP4(Ala) transfection was found to abolish HG-induced hyperpermeability, resulting in a 1.46-fold decrease in dextran leakage, and a 1.62-fold elevation in TER as compared to HG treatment group. To the contrary, transduction with MAP4(Glu) resulted in higher hyperpermeability (1.36-fold elevation in dextran leakage and 2.58-fold decrease in TER as compared to HG group) (Figure. 7A-B). Using TUNEL staining, a rise in apoptosis was detected in HG group, the effect of which was ameliorated and accentuated by MAP4(Ala) and MAP4(Glu) transfection, respectively (Figure. 7C-D). Given the role of MAP4 in MT dynamic regulation, and that cytoskeleton possesses an essential effect in maintaining the integrity of podocytes foot processes, we determined whether MAP4 phosphorylation regulates permeability though cytoskeleton rearrangement. Scrutiny of F-actin and MT distribution in podocytes revealed that HG treatment induced the depolymerization of $\mathrm{MT}$, the stress fiber reorganization of F-actin, and the re-distribution of F-actin from the perinuclear region towards the periphery of cells. MAP4(Glu) transfection accentuated while MAP4(Ala) transfection mitigated these responses (Figure. 7E).

\section{p38/MAPK activation mediated MAP4 phosphorylation induced podocyte EMT and apoptosis}

Our previous study indicated that p38/MAPK serves as a crucial kinase modulating MAP4 phosphorylation in hypoxic cardiomyocytes (Hu et al., 2010). Here we assessed whether p38/MAPK was involved in $\mathrm{HG}$-induced MAP4 phosphorylation in podocytes. Our findings revealed elevated expressions of phosphorylation of MAP4 (S737 and S760) and P38 following HG stimulation (without affecting MAP4 and p38). In contrast, SB203580 (a p38/MAPK inhibitor) suppressed HG-induced p38/MAPK and MAP4 phosphorylation (S737 and S760) (Figure. 8A and Supplementary Figure 1). These findings inferred that $\mathrm{p38/MAPK}$ was a key upstream kinase to promote phosphorylated MAP4 under HG challenge.

We next discern the effect of p38/MAPK in HG-mediated podocyte EMT, hyperpermeability and apoptosis. Podocytes were transfected with MKK6(Glu) adenovirus to activate p38/MAPK, while pretreatment of SB203580 inhibited HG-induced p38/MAPK activation. WB analysis indicated that MKK6(Glu) aggravated while SB203580 alleviated HG-induced reduction of epithelial markers and elevation of 
mesenchymal markers in podocytes (Figure. 8B-C). Immunofluorescence staining of podocytes revealed similar pattern in WT-1, Nephrin, FSP1 and Desmin (Figure. 8D-E). In addition, SB203580 pretreatment protected against $\mathrm{HG}$-induced podocytes hyperpermability and apoptosis, while the problems were aggravated after MKK6(Glu) transfection (Figure. 9A-D). Furthermore, MKK6(Glu) transfection accentuated while SB203580 mitigated HG-induced changes in cytoskeleton (Figure. 9E).

Then we assessed the effect of MAP4 phosphorylation in p38/MAPK-mediated cytoskeleton rearrangement. Podocytes were transfected with MKK6(Glu), MAP4(Ala) or both. Our data revealed that podocytes overexpressing MAP4(Ala) were more resistant to MKK6(Glu) transfection compared with the CMV-null control on cytoskeleton rearrangement (Figure. 9F). These data denoted the role of p38/MAPK as an upstream regulator for MAP4 phosphorylation and further demonstrated a crucial role for MAP4 phosphorylation in p38/MAPK-induced podocyte EMT, hyperpermeability and apoptosis.

\section{Discussion}

Salient findings from current study indicated that MAP4, the classical skeletal protein, were phosphorylated in urine of diabetic patients and the kidney of the STZ-induced diabetic mice, which was accompanied with glomerular proteinuria associated with nephropathy. The MAP4 KI mice, a mouse model of hyperphosphorylated MAP4 at S737 and S760, triggered proteinuria with aging, accompanied with increased EMT and apoptosis of podocytes. Additionally, MAP4 KI mice were much more susceptible to STZ treatment and showed robust DN pathology as compared to WT mice. In cultured podocytes, MAP4 phosphorylation was universal increased following hyperglycemia as the down-stream effector of p38/MAPK, which led to podocytes undergoing functional, morphological, and molecular changes reminiscent of apoptosis and EMT through induced cytoskeleton rearrangement. Otherwise, it should be indicated that the MAP4 KI mice also developed retinopathy. The diabetic retinopathy and DN are the main microvascular complications of diabetes, and the co-occurrence of retinopathy and nephropathy in MAP4 KI mice suggest that the MAP4 phosphorylation may act as the common mechanism mediating the microvascular disease in diabetes.

Podocyte loss and dysfunction are known to possess a crucial effect in the pathogenesis of proteinuria and glomerulosclerosis (Daehn et al., 2014). Podocytes are terminally differentiated cells which cover GBM over glomerular capillary. Podocytes maintain glomerular structural integrity through GBM counteracting outward expansion, for which the foot processes is the major functional unit (Garg, 2018). When subject to pathological challenge, podocytes undergo a series of changes, involving EMT, dissociation, hypertrophy, and apoptosis, relying on the degree of injury (Dai and Liu, 2017; Ling et al., 2018; Wang et al., 2018). Since their limited ability of proliferation, podocyte dissociation from GBM and apoptosis will unavoidably causing cell loss or drop out, which decrease density of podocyte, leading to impaired glomerular filtration, and ultimately proteinuria. Recent experimental data demonstrated that podocyte loss often occurs in late stage of chronic kidney disease in which proteinuria is already distinct (Dai et al., 2006; El-Aouni et al., 2006). It is thus plausible to infer that the abnormal modulation of 
podocyte function or differentiation, rather than podocyte loss, might be a trigger of proteinuria in various clinical conditions.

EMT of podocytes is characterized by deficiency of epithelial markers, including P-cadherin, ZO-1, and Nephrin, and de novo gain classic mesenchymal markers, such as collagen I, FSP1, Desmin and fibronectin (Kumar et al., 2016). As the crucial part of the slit diaphragm cell adhesion complexes, loss of Nephrin will contribute to podocyte dissociation from GBM and disrupt the integrity of slit diaphragm (Asanuma and Mundel, 2003). EMT has recently been observed in STZ-treated diabetic rats (Du et al., 2017), and exists when subjected to hypoxia, reactive oxygen species, advanced glycation end products and numerous pro-fibrotic cytokines, growth factors and metalloproteinases (Wei and Yang, 2016).

One interesting finding from the present study is the profound apoptosis and EMT of podocytes in response to MAP4 hyperphosphorylation in STZ-treated and MAP4 KI mice. An essential feature that distinguishes podocytes from other renal cells is that they are terminally differentiated, and have no proliferative ability when subject to injury or noxious stimuli. Cell proliferation rate seen in our study exhibited little difference among WT, STZ-treated and MAP4 KI mice, which suggested that increased mesenchymal cells were possibly attributed to the development of podocyte EMT rather than mesangial cell proliferation. In spite of this, many questions remain unknown such as if increased mesenchymal cells originated from development EMT of endothelial cells, whether proteinuria was also attributed to overt apoptosis or EMT of podocytes.

Cytoskeleton rearrangement is crucial to accomplish EMT. It is not only the default consequence of EMT activation, but also a regulator in this process (Shankar et al., 2010; Li et al., 2011). It has been reported that actin reorganization is tightly connected to foot processes effacement related to proteinuria (Hu et al., 2017). However, the precise mechanism regulating the cytoskeleton rearrangement when podocytes undergo EMT is still unclear and needs further investigation. Interactions between MT and actin represent a phenomenon governing a number of basic cellular processes, such as cell motility and cell division. MAPs has been shown to be involved in regulating the interactions of MT and actin in the formation of cytoskeletal network (Farias et al., 2002). In this study, we noted overtly elevated phosphorylation of MAP4 in STZ-induced diabetic mice. On the other hand, mutated mouse model with hyperphosphorylated MAP4 (S737E and S760E) displayed proteinuria with aging, accompanied with prominent EMT of podocytes, in a manner reminiscent of the pathologic changes in the DN, furthermore, STZ stimulation aggravated such kidney pathological changes in MAP4 KI mice as compared to WT littermates. It is an interesting phenomenon in the present study that both aging and STZ treatment are involved in DN in our present study. In MAP4 KI- and STZ-induced mice, nephropathy progress overtime is the result of accumulated damage induced by MAP4 hyperphosphorylation or high glucose. Age is not the direct cause, but aged organ/cells have less compensating ability. Thus, we observed worse diabetic nephropathy phenotype in aged MAP4 KI mice. In all, aging and accumulated damages induced by MAP4 hyperphosphorylation and high glucose are both conditions making MAP4 KI mice harder to keep their in vivo homeostasis. In addition, in vitro data revealed that HG promoted MAP4 hyperphosphorylation, and either HG or aberrant regulation of MAP4 phosphorylation led to cytoskeleton and F-actin rearrangement 
and impaired filtration barrier function in podocytes. MAP4(Ala) mutant, which mimicked dephosphorylated MAP4, suppressed EMT and alleviated increased cell permeability. Therefore, these in vivo and in vitro observations favored a novel insight for MAP4 in modulating podocyte EMT and proteinuria in the development of DN through MAP4 phosphorylation.

In our previous study, p38/MAPK activation was sufficient to trigger MAP4 phosphorylation in cardiomyocytes (Hu et al., 2014). Among many factors implicated in the pathogenesis of DN, p38/MAPK serves as one of critical mediators for inflammatory reactions and mitochondrial malfunction in diabetes (Liu et al., 2016). Activated p38/MAPK was found in renal proximal tubular epithelial cells (PTECs) and contributes to EMT (Zhang et al., 2015). Aberrant p38 phosphorylation was also correlated with podocyte cytoskeletal dynamics (Hu et al., 2017). In our hands, inhibition of p38/MAPK was proved to alleviate MT, F-actin arrangement and hyperpermeability of podocytes in response to HG challenge accompanied with decreased MAP4 phosphorylation. Our data are the first, to our best knowledge, to suggest a novel role for p38/MAPK in regulating podocyte EMT through phosphorylating MAP4 in DN. Our earlier studies depicted a role for MAP4 phosphorylation in endogenous apoptosis in cardiomyocytes in response to hypoxia. Whether phosphorylated MAP4 mediates podocyte apoptosis through similar mechanism warrants further scrutiny.

\section{Conclusions}

Here in the present study using a hyperphosphorylated MAP4 mouse strain, we showed that MAP4 phosphorylation induced MT and F-actin rearrangement, podocyte EMT and apoptosis, leading to proteinuria, in a manner reminiscent of DN. Blockade of p38/MAPK signaling prevented the dedifferentiation and apoptosis of podocytes. These data implied that manipulating p38/MAPK-MAP4 phosphorylation signaling may unearth a future therapeutic target to alleviate proteinuria and renal fibrosis in DN.

\section{Abbreviations}

DN

Diabetic nephropathy

MAP4

microtubule associated protein 4

p-MAP4

MAP4 phosphorylation

STZ

streptozocin

EMT

epithelial-to-mesenchymal transition

HG

high glucose 
MAPS

Microtubule-associated proteins

MT

microtubule

MKK6

mitogen-activated protein kinase kinase 6

MAP4 KI

MAP4 (S737/760E) knock-in

CR

urine creatinine

UN

urea nitrogen, Cys-c:cystatin c

TEM

transmission electron microscope

GBM

glomerular basement membrane

WT-1

Wilms Tumor protein

FSP1

fibroblast-specific protein 1

TUNEL

terminal deoxynucleotidyl transferase-mediated dUTP nick-end labeling

EdU

5-ethynyl-2'-deoxyuridine

ELISA

Enzyme-Linked Immunosorbent Assay

TER

Measurement of transepithelial electric resistance

FITC

fluorescein isothiocyanate

ANOVA

one-way analysis of variance

PTECs

proximal tubular epithelial cells.

\section{Declarations}

Ethical Approval and Consent to participate

Ethics statement of patient urine samples 
The Ethics Committee of Southwest Hospital, Third Military Medical University (Army Medical University) approved the present study (approval number: KY2020085). Prior to be included in the study, all participants signed relevant informed consent. Participants were identified using a number, not their name. It was conducted in adherence with the Declaration of Helsinki. All methods were carried out in accordance with the institutional guidelines of the ethics committee at Southwest Hospital, Third Military Medical University (Army Medical University) (Chongqing, China).

\section{Ethics statement of animal study}

All animal experiments were implemented in accordance with the Guide for the Care and Use of Laboratory Animals announced by the US National Institutes of Health (NIH Publication, 8th Edition, 2011) and were granted permission by the Animal Experiment Ethics Committee of the Army Medical University (the Third Military Medical University).

\section{Consent for publication}

Not applicable.

\section{Availability of supporting data}

Not applicable.

\section{Competing interests}

All authors claim to have no conflicts of interest.

\section{Funding}

This was supported by the State Key Laboratory of Trauma, Burns and Combined Injury Research Foundation (No. SKLYQ201802), and the National Youth Fund Project of China (No. 82003323), and the Military Medical Innovation Program in Daping Hospital (2019CXJSC014).

\section{Authors' contributions}

G.L., J. H. and Y.H. supervised the work, L.L. and Y.F. designed the experiments with help from Q. Z., J.Z., J.R., C.S., X. L. and S.L., L.L. and Y.F. performed the experiments with help from Q. Z., J.Z., J.R., C.S., and S.L., L.L. and Y.F. analyzed the data with help from C.S. and S.L, L.L., Y.F. and J.R. co-wrote the manuscript. All authors discussed the results and commented on the manuscript. All authors read and approved the final manuscript.

\section{Acknowledgements}

Not applicable.

\section{Authors' information}


${ }^{1}$ Institute of Burn Research, Southwest Hospital, Third Military Medical University (Army Medical University), Chongqing, China, ${ }^{2}$ State Key Laboratory of Trauma, Burns and Combined Injury, Southwest Hospital, Third Military Medical University (Army Medical University), Chongqing, China, ${ }^{3}$ Department of Dermatology, Daping Hospital, Third Military Medical University (Army Medical University), Chongqing, China, ${ }^{4}$ Department of Cardiology, Shanghai Institute of Cardiovascular Diseases, Zhongshan Hospital, Fudan University, Shanghai 200032, China. ${ }^{5}$ Department of Laboratory Medicine and Pathology, University of Washington, Seattle, WA 98195, USA. ${ }^{6}$ Department of Ophthalmology, Southwest Hospital, Third Military Medical University (Army Medical University), Chongqing, China, ${ }^{7}$ The Second Affiliated Hospital of Chongqing Medical University, Chongqing, China, ${ }^{8}$ Endocrinology Department, Southwest Hospital, Third Military Medical University (Army Medical University), Chongqing, China. ${ }^{9}$ Department of Wound Repair, Institute of Wound Repair, Shenzhen People's Hospital,First Affiliated Hospital of Southern University of Science and Technology, Shenzhen, China.

\section{References}

1. Asanuma, K., Kim, K., Oh, J., Giardino, L., Chabanis, S., Faul, C., et al. (2005). Synaptopodin regulates the actin-bundling activity of alpha-actinin in an isoform-specific manner. J Clin Invest 115(5), 11881198. doi: 10.1172/jci23371.

2. Asanuma, K., and Mundel, P. (2003). The role of podocytes in glomerular pathobiology. Clin Exp Nephrol 7(4), 255-259. doi: 10.1007/s10157-003-0259-6.

3. Chen, W., Jiang, Y., Han, J., Hu, J., He, T., Yan, T., et al. (2017). Atgl deficiency induces podocyte apoptosis and leads to glomerular filtration barrier damage. Febs j 284(7), 1070-1081. doi: $10.1111 /$ febs. 14038 .

4. Czajka, A., Ajaz, S., Gnudi, L., Parsade, C.K., Jones, P., Reid, F., et al. (2015). Altered Mitochondrial Function, Mitochondrial DNA and Reduced Metabolic Flexibility in Patients With Diabetic Nephropathy. EBioMedicine 2(6), 499-512. doi: 10.1016/j.ebiom.2015.04.002.

5. Daehn, I., Casalena, G., Zhang, T., Shi, S., Fenninger, F., Barasch, N., et al. (2014). Endothelial mitochondrial oxidative stress determines podocyte depletion in segmental glomerulosclerosis. $J$ Clin Invest 124(4), 1608-1621. doi: 10.1172/jci71195.

6. Dai, C., Stolz, D.B., Bastacky, S.I., St-Arnaud, R., Wu, C., Dedhar, S., et al. (2006). Essential role of integrin-linked kinase in podocyte biology: Bridging the integrin and slit diaphragm signaling. $J \mathrm{Am}$ Soc Nephrol 17(8), 2164-2175. doi: 10.1681/asn.2006010033.

7. Dai, H., and Liu, Q. (2017). Research Progress on Mechanism of Podocyte Depletion in Diabetic Nephropathy. J Diabetes Res 2017, 2615286. doi: 10.1155/2017/2615286.

8. Drewes, G., Ebneth, A., and Mandelkow, E.M. (1998). MAPs, MARKs and microtubule dynamics. Trends Biochem Sci 23(8), 307-311.

9. Du, L., Hao, M., Li, C., Wu, W., Wang, W., Ma, Z., et al. (2017). Quercetin inhibited epithelial mesenchymal transition in diabetic rats, high-glucose-cultured lens, and SRA01/04 cells through 
transforming growth factor-beta2/phosphoinositide 3-kinase/Akt pathway. Mol Cell Endocrinol 452, 44-56. doi: 10.1016/j.mce.2017.05.011.

10. Ebneth, A., Drewes, G., Mandelkow, E.M., and Mandelkow, E. (1999). Phosphorylation of MAP2c and MAP4 by MARK kinases leads to the destabilization of microtubules in cells. Cell Motil Cytoskeleton 44(3), 209-224. doi: 10.1002/(SICI)1097-0169(199911)44:3<209::AID-CM6>3.0.C0,2-4.

11. El-Aouni, C., Herbach, N., Blattner, S.M., Henger, A., Rastaldi, M.P., Jarad, G., et al. (2006). Podocytespecific deletion of integrin-linked kinase results in severe glomerular basement membrane alterations and progressive glomerulosclerosis. J Am Soc Nephro/ 17(5), 1334-1344. doi: 10.1681/asn.2005090921.

12. Farias, G.A., Munoz, J.P., Garrido, J., and Maccioni, R.B. (2002). Tubulin, actin, and tau protein interactions and the study of their macromolecular assemblies. J Cell Biochem 85(2), 315-324.

13. Gallagher, H., and Suckling, R.J. (2016). Diabetic nephropathy: where are we on the journey from pathophysiology to treatment? Diabetes Obes Metab 18(7), 641-647. doi: 10.1111/dom.12630.

14. Garg, P. (2018). A Review of Podocyte Biology. Am J Nephrol 47 Suppl 1, 3-13. doi: $10.1159 / 000481633$.

15. Hu, J., Chu, Z., Han, J., Zhang, Q., Zhang, D., Dang, Y., et al. (2014). Phosphorylation-dependent mitochondrial translocation of MAP4 is an early step in hypoxia-induced apoptosis in cardiomyocytes. Cell Death Dis 5, e1424. doi: 10.1038/cddis.2014.369.

16. Hu, J.Y., Chu, Z.G., Han, J., Dang, Y.M., Yan, H., Zhang, Q., et al. (2010). The p38/MAPK pathway regulates microtubule polymerization through phosphorylation of MAP4 and Op18 in hypoxic cells. Cell Mol Life Sci 67(2), 321-333. doi: 10.1007/s00018-009-0187-z.

17. Hu, M., Fan, M., Zhen, J., Lin, J., Wang, Q., Lv, Z., et al. (2017). FAK contributes to proteinuria in hypercholesterolaemic rats and modulates podocyte F-actin re-organization via activating p38 in response to ox-LDL. J Cell Mol Med 21(3), 552-567. doi: 10.1111/jcmm.13001.

18. Illenberger, S., Drewes, G., Trinczek, B., Biernat, J., Meyer, H.E., Olmsted, J.B., et al. (1996). Phosphorylation of microtubule-associated proteins MAP2 and MAP4 by the protein kinase p110mark. Phosphorylation sites and regulation of microtubule dynamics. J Biol Chem 271(18), 10834-10843.

19. Kang, Y.S., Li, Y., Dai, C., Kiss, L.P., Wu, C., and Liu, Y. (2010). Inhibition of integrin-linked kinase blocks podocyte epithelial-mesenchymal transition and ameliorates proteinuria. Kidney Int 78(4), 363-373. doi: 10.1038/ki.2010.137.

20. Kitazawa, H., lida, J., Uchida, A., Haino-Fukushima, K., Itoh, T.J., Hotani, H., et al. (2000). Ser787 in the proline-rich region of human MAP4 is a critical phosphorylation site that reduces its activity to promote tubulin polymerization. Cell Struct Funct 25(1), 33-39.

21. Kumar, P.A., Welsh, G.I., Raghu, G., Menon, R.K., Saleem, M.A., and Reddy, G.B. (2016). Carboxymethyl lysine induces EMT in podocytes through transcription factor ZEB2: Implications for podocyte depletion and proteinuria in diabetes mellitus. Arch Biochem Biophys 590, 10-19. doi: 10.1016/j.abb.2015.11.003. 
22. Li, L., Hu, J., He, T., Zhang, Q., Yang, X., Lan, X., et al. (2015). P38/MAPK contributes to endothelial barrier dysfunction via MAP4 phosphorylation-dependent microtubule disassembly in inflammationinduced acute lung injury. Sci Rep 5, 8895. doi: 10.1038/srep08895.

23. Li, L., Zhang, Q., Zhang, X., Zhang, J., Wang, X., Ren, J., et al. (2018). Microtubule associated protein 4 phosphorylation leads to pathological cardiac remodeling in mice. EBioMedicine 37, 221-235. doi: 10.1016/j.ebiom.2018.10.017.

24. Li, N., Jiang, P., Du, W., Wu, Z., Li, C., Qiao, M., et al. (2011). Siva1 suppresses epithelial-mesenchymal transition and metastasis of tumor cells by inhibiting stathmin and stabilizing microtubules. Proc Natl Acad Sci U S A 108(31), 12851-12856. doi: 10.1073/pnas.1017372108.

25. Ling, L., Tan, Z., Zhang, C., Gui, S., Hu, Y., and Chen, L. (2018). Long noncoding RNA ENSRNOG00000037522 is involved in the podocyte epithelialmesenchymal transition in diabetic rats. Int J Mol Med 41 (5), 2704-2714. doi: 10.3892/ijmm.2018.3457.

26. Liu, W.T., Peng, F.F., Li, H.Y., Chen, X.W., Gong, W.Q., Chen, W.J., et al. (2016). Metadherin facilitates podocyte apoptosis in diabetic nephropathy. Cell Death Dis 7(11), e2477. doi:

10.1038/cddis.2016.335.

27. Locatelli, F., Pozzoni, P., and Del Vecchio, L. (2004). Renal replacement therapy in patients with diabetes and end-stage renal disease. J Am Soc Nephrol 15 Suppl 1, S25-29.

28. Najafian, B., Basgen, J.M., and Mauer, M. (2002). Estimating mean glomerular volume using two arbitrary parallel sections. J Am Soc Nephro/ 13(11), 2697-2705.

29. Raingeaud, J., Whitmarsh, A.J., Barrett, T., Derijard, B., and Davis, R.J. (1996). MKK3- and MKK6regulated gene expression is mediated by the p38 mitogen-activated protein kinase signal transduction pathway. Mol Cell Biol 16(3), 1247-1255.

30. Rask-Madsen, C., and King, G.L. (2010). Diabetes: Podocytes lose their footing. Nature 468(7320), 4244. doi: $10.1038 / 468042 a$.

31. Shankar, J., Messenberg, A., Chan, J., Underhill, T.M., Foster, L.J., and Nabi, I.R. (2010). Pseudopodial actin dynamics control epithelial-mesenchymal transition in metastatic cancer cells. Cancer Res 70(9), 3780-3790. doi: 10.1158/0008-5472.can-09-4439.

32. Shankland, S.J., Pippin, J.W., Reiser, J., and Mundel, P. (2007). Podocytes in culture: past, present, and future. Kidney Int 72(1), 26-36. doi: 10.1038/sj.ki.5002291.

33. Wang, R.M., Wang, Z.B., Wang, Y., Liu, W.Y., Li, Y., Tong, L.C., et al. (2018). Swiprosin-1 Promotes Mitochondria-Dependent Apoptosis of Glomerular Podocytes via P38 MAPK Pathway in Early-Stage Diabetic Nephropathy. Cell Physiol Biochem 45(3), 899-916. doi: 10.1159/000487285.

34. Wei, S.C., and Yang, J. (2016). Forcing through Tumor Metastasis: The Interplay between Tissue Rigidity and Epithelial-Mesenchymal Transition. Trends Cell Biol 26(2), 111-120. doi: 10.1016/j.tcb.2015.09.009.

35. Ying, Q., and Wu, G. (2017). Molecular mechanisms involved in podocyte EMT and concomitant diabetic kidney diseases: an update. Ren Fail 39(1), 474-483. doi: 10.1080/0886022x.2017.1313164. 
36. Zhang, X., Liang, D., Chi, Z.H., Chu, Q., Zhao, C., Ma, R.Z., et al. (2015). Effect of zinc on high glucoseinduced epithelial-to-mesenchymal transition in renal tubular epithelial cells. Int J Mol Med 35(6), 1747-1754. doi: 10.3892/ijmm.2015.2170.

37. Zurbig, P., Jerums, G., Hovind, P., Macisaac, R.J., Mischak, H., Nielsen, S.E., et al. (2012). Urinary proteomics for early diagnosis in diabetic nephropathy. Diabetes 61(12), 3304-3313. doi: $10.2337 / \mathrm{db} 12-0348$.

\section{Figures}
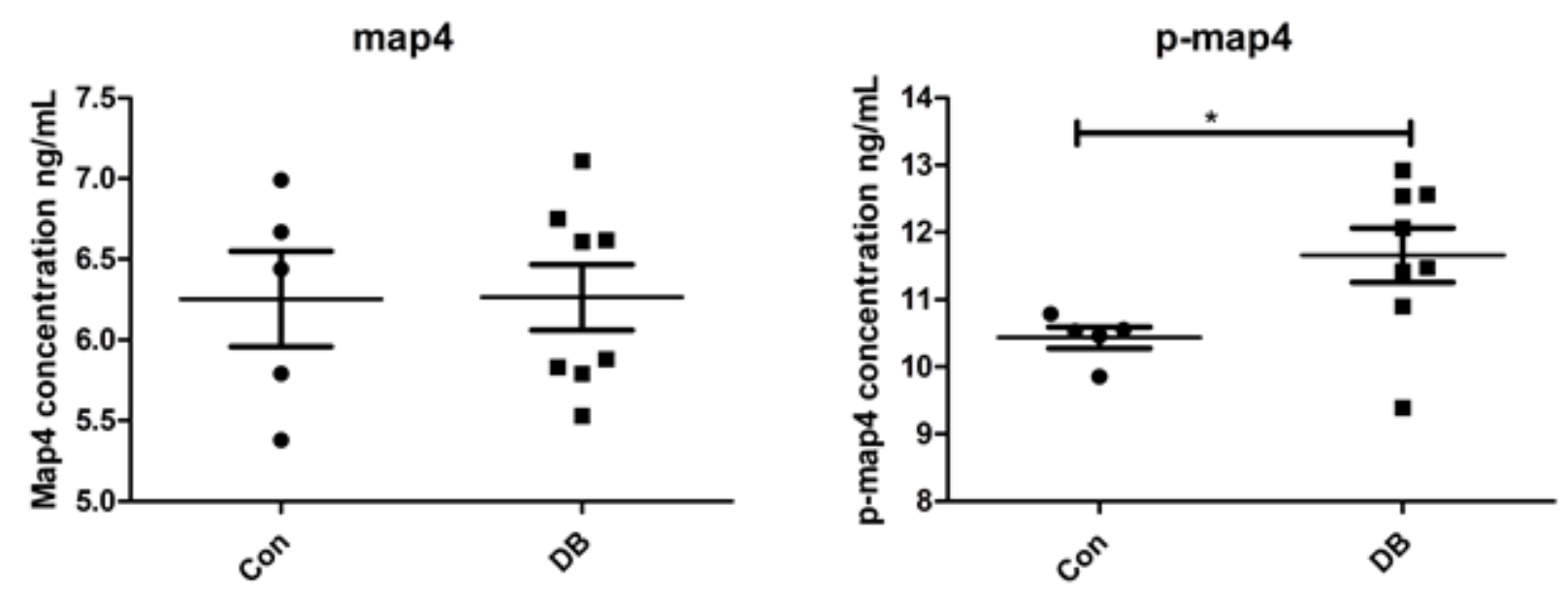

Figure 1

MAP4 phosphorylation increased in urine samples of diabetic patients

Concentrations of MAP4 (A) and p-MAP4 (B) in urine samples were measured by ELISA. $n=5$ or $8 .{ }^{*} P<$ 0.05 when compared with control group. $P$ value were derived from two-tailed Student's $t$-test.

Figure 2

Nephritic MAP4 hyperphosphorylation along with proteinuria in DN. (A, B) Body weight (A) and random blood glucose (B) was assessed between WT and WT STZ mice. $n=8$. (C, D) Representative WB $(C)$ and quantitative analysis (D) depicting p-M (S737 and S760) in WT and WT STZ littermates. $n=6$. p-M, p- 
MAP4. (E-H) UACR, serum levels of CR, UN and Cys-c were detected by commercial kits. $n=8$. (I, J) HE staining and glomerular volume quantitative analysis of deparaffinized kidney tissue section between WT and WT STZ mice. Bar, $20 \mu \mathrm{m} . \mathrm{n}=6$. (K) Masson's trichrome staining of WT and WT STZ mice. Bar, 20 $\mu \mathrm{m} . \mathrm{n}=6$. $(\mathrm{L}, \mathrm{M})$ Quantitative analysis of foot processes fusion $(L)$ and TEM observation $(M)$. Bar, $5 \mu \mathrm{m}$. $n=6$. $(N, 0)$ Representative confocal immunofluorescence images and quantitative analysis showing the epithelial and mesenchymal cell markers of frozen kidney tissue section. Bar, 10 or $25 \mu \mathrm{m} . n=6 .(P, Q)$ Podocyte apoptosis (white arrows) of frozen kidney tissue section was detected by WT-1 and TUNEL costaining. Bar, $10 \mu \mathrm{m} . \mathrm{n}=6$. $(\mathrm{R}, \mathrm{S})$ Cell proliferation (white arrows) of frozen kidney tissue section was determined by EdU staining. Bar, $10 \mu \mathrm{m} . \mathrm{n}=6$. The graph showed mean $\pm \mathrm{SD} .{ }^{\star} P<0.05,{ }^{\star \star} P<0.01$, ${ }^{\star \star \star} P<$ 0.001 . $P$ values were derived from two-tailed Student's $t$-test.

\section{Figure 3}

\section{MAP4 hyperphosphorylation mutation leads to proteinuria and nephropathy reminiscent of diabetic} kidney disease in MAP4 KI mice. (A, B) Representative WB (A) and quantitative analysis (B) depicting p-M (S737 and S760) in WT and MAP4 KI mice at 10-14 weeks. $n=6$. (C-F) UACR, serum levels of CR, UN and Cys-c were determined in two mouse models at different ages. $n=8$. (G, H) HE and Masson's trichrome staining as well as glomerular volume quantitative analysis of deparaffinized kidney tissue section was examined at different ages. Bar, $20 \mu \mathrm{m} . n=6$. $(\mathrm{I}, \mathrm{J})$ Quantitative analysis of foot processes fusion $(\mathrm{I})$ and TEM observation $(J)$. Bar, $2 \mu \mathrm{m} . n=6$. $(K, L)$ Podocyte apoptosis of frozen kidney tissue section was detected by WT-1 and TUNEL co-staining. Bar, $10 \mu \mathrm{m} . \mathrm{n}=6$. $(\mathrm{M}, \mathrm{N})$ Cell proliferation of frozen kidney tissue section was determined by EdU staining. Bar, $25 \mu \mathrm{m} . \mathrm{n}=6$. The graph showed mean $\pm \mathrm{SD}$. ${ }^{*} P<$ $0.05,{ }^{\star} P<0.01, \star \star \star P<0.001$. $P$ values were derived from one-way ANOVA with Bonferroni's post-test.

\section{Figure 4}

\section{MAP4 hyperphosphorylation mutation leads to EMT and diabetic retinopathy in MAP4 KI mice. (A, B)} Representative confocal immunofluorescence images $(A)$ and quantitative analysis (B) showing the epithelial and mesenchymal cell markers of frozen kidney section at different ages. Bar, 10 or $25 \mu \mathrm{m} . \mathrm{n}=$ 6. (C) Fluorescein angiogram showing retinal vasculature of (a) normal control mouse (c) STZ-induced diabetic mouse (e) MAP4 KI mouse (g) STZ treated MAP4 KI mouse. The (b), (d), (f) and (h) are the represented enlarged area of the (a), (c), (e) and (g). Arrow points to the microaneurysms. ${ }^{\star} P<0.05, \star \star \star P$ $<0.001$. $P$ values were derived from one-way ANOVA with Bonferroni's post-test. 
Detection of proteinuria and nephropathy reminiscent of diabetic kidney disease in STZ-induced WT and MAP4 KI mice. (A-D) UACR, serum levels of CR, UN and Cys-c were determined in two mouse models at 10-14 weeks after STZ treatment. $n=8$. $(E, F)$ Quantitative analysis of foot processes fusion and TEM observation in WT and MAP4 KI mice at 10-14 weeks after STZ treatment. Bar, $2 \mu \mathrm{m} . \mathrm{n}=6 .(\mathbf{G}, \mathrm{H})$ Representative confocal immunofluorescence images and quantitative analysis showing the epithelial and mesenchymal cell markers of frozen kidney section in two mouse models at 10-14 weeks after STZ treatment. Bar, 10 or $25 \mu \mathrm{m} . \mathrm{n}=6$. Data were shown as mean $\pm \mathrm{SEM}$. ${ }^{\star} P<0.05$. $P$ values were derived from two-tailed Student's $t$-test.

\section{Figure 6}

MAP4 phosphorylation induced EMT in podocyte. (A) Identification of adenovirus efficacy of MAP4(Ala) or MAP4(Glu). (B, C) Representative WB (B) and quantitative analysis (C) showing the epithelial and mesenchymal cell markers of podocyte after MAP4(Ala) or MAP4(Glu) transfection with or without HG stimulation. (D, E) Representative confocal immunofluorescence images (D) and quantitative analysis (E) showing the epithelial and mesenchymal cell markers of podocyte after MAP4(Ala) or MAP4(Glu) transfection with or without HG stimulation. Bar, $25 \mu \mathrm{m}$. MPC5, cell line of mouse podocyte. Data were shown as mean \pm SEM. The experiment was conducted 6 times. ${ }^{\star} P<0.05,{ }^{\star} * P<0.01,{ }^{\star} * \star P<0.001 . P$ values were derived from one-way ANOVA with Bonferroni's post-test.

\section{Figure 7}

\section{MAP4 phosphorylation induced cytoskeleton rearrangement and apoptosis in podocyte. (A, B) The} permeability of podocyte was assessed by measuring the influx of FITC-conjugated dextran and the TER across the cells. (C, D) Podocyte apoptosis was detected by TUNEL staining. Bar, $25 \mu \mathrm{m}$. (E) Representative confocal immunofluorescence images showing the MTs and F-actin organization of the podocytes after MAP4(Ala) or MAP4(Glu) transfection with or without HG stimulation. Bar, $10 \mu \mathrm{m}$. The inserts showed high magnification images of the MT and F-actin network. Data were shown as mean \pm SEM. The experiment was conducted 6 times. ${ }^{\star} P<0.05,{ }^{\star} * P<0.01,{ }^{\star} * \star P<0.001 . P$ values were derived from one-way ANOVA with Bonferroni's post-test.

\section{Figure 8}

p38/MAPK activation mediated HG-induced podocyte EMT. (A) Detection of p-M (S737 and S760) and pP38 with or without SB203580 (SB) and MKK6(Glu) transfection. (B, C) WB analysis showing the epithelial and mesenchymal cell markers of podocyte with or without SB or MKK6(Glu) transfection. (D, E) 
Representative confocal immunofluorescence images (D) and quantitative analysis (E) showing the epithelial and mesenchymal cell markers of podocyte. Bar, $25 \mu \mathrm{m}$. Data were shown as mean $\pm \mathrm{SEM}$. The experiment was conducted 6 times. ${ }^{*} P<0.05,{ }^{*} P<0.01,{ }^{\star} * \star P<0.001$. $P$ values were derived from oneway ANOVA with Bonferroni's post-test.

\section{Figure 9}

p38/MAPK activation mediated HG-induced podocyte apoptosis and cytoskeleton. (A, B) The permeability of podocyte was assessed by measuring the influx of FITC-conjugated dextran and the TER across the cells. (C, D) TUNEL staining showed podocyte apoptosis. Bar, $25 \mu \mathrm{m}$. (E) Representative confocal immunofluorescence images showing the MTs and F-actin organization of the podocytes. Bar, $10 \mu \mathrm{m}$. The inserts showed high magnification images of the MT and F-actin network. (F) Representative immunofluorescence images showing the MTs and F-actin organization of the podocyte after the cells were transiently transfected with MAP4(Ala), MKK6(Glu) or both. Bar, $10 \mu \mathrm{m}$. The inserts showed high magnification images of the peripheral MT network. Data were shown as mean \pm SEM. The experiment was conducted 6 times. ${ }^{\star} P<0.05,{ }^{*} P<0.01,{ }^{\star * \star} P<0.001$. $P$ values were derived from one-way ANOVA with Bonferroni's post-test.

\section{Supplementary Files}

This is a list of supplementary files associated with this preprint. Click to download.

- SupplementaryFigure1.tif 